\title{
Imaging Blood Vessel Morphology in Skin: Dynamic Optical Coherence Tomography as a Novel Potential Diagnostic Tool in Dermatology
}

\author{
Sandra Schuh · Jon Holmes · Martina Ulrich • Lotte Themstrup • \\ Gregor B. E. Jemec · Nathalie De Carvalho · Giovanni Pellacani • \\ Julia Welzel
}

Received: November 23, 2016 / Published online: March 3, 2017

(C) The Author(s) 2017. This article is published with open access at Springerlink.com

\begin{abstract}
Conventional optical coherence tomography (OCT) enables the visualization of morphological changes of skin cancer. The use of OCT in the diagnostic investigation and in the therapy decision of non-melanoma skin cancer and other skin changes is already established, and has found its way into routine practice. With the development of speckle-variance OCT, also named dynamic OCT (D-OCT), the vascular architecture and the blood flow of the skin can be displayed in vivo and in 3D. This novel angiographic variant of OCT offers the ability to
\end{abstract}

Enhanced content To view enhanced content for this article go to http://www.medengine.com/Redeem/ 8F87F06017F44BCF.

S. Schuh $\cdot$ J. Welzel $(\bowtie)$

Department of Dermatology, General Hospital

Augsburg, Augsburg, Germany

e-mail: Julia.Welzel@klinikum-augsburg.de

J. Holmes

Michelson Diagnostics Ltd., Maidstone, UK

M. Ulrich

CMB Collegium Medicum Berlin, Berlin, Germany

L. Themstrup - G. B. E. Jemec

Department of Dermatology, Zealand University

Hospital, Roskilde, Denmark

N. De Carvalho · G. Pellacani

Department of Dermatology, University of Modena and Reggio Emilia, Modena, Italy visualize and measure vessel morphology providing a new insight into healthy, inflammatory and neoplastic skin lesions such as malignant melanoma. This review focuses on the possibilities of using D-OCT on healthy and diseased skin. We suggest and illustrate key diagnostic characteristics by analyzing the initial publications and preliminary unpublished data on vessel morphology and distribution. The potential of D-OCT as a diagnostic tool in dermatology is examined and may give rise to future studies on D-OCT, which are needed to confirm the aforementioned features.

Keywords: Degenerative skin diseases; Dynamic optical coherence tomography; Healthy skin; Imaging method; Inflammatory skin diseases; Malignant melanoma; Noninvasive diagnosis; Non-melanoma skin cancer; Skin blood vessels; Treatment effects

\section{INTRODUCTION}

Optical coherence tomography (OCT) is a noninvasive imaging technique that has been applied to skin since 1997 [1]. It provides two-dimensional cross-sectional and horizontal images of skin areas covering several square millimeters with a penetration depth of up to $1.5 \mathrm{~mm}$. The OCT images are displayed in real time, showing architectural changes of the skin 
layers. In most cases, the resolution of $3-15 \mu \mathrm{m}$ is not high enough to distinguish single cells.

In dermatology, conventional OCT is mainly used and is already well established in the diagnosis of epithelial skin cancer such as basal cell carcinomas with good sensitivity (96\%) and specificity (75\%) [2]. Basal cell carcinomas show typical features such as ovoid 'nests' with a dark rim, and can be easily differentiated from actinic keratoses. Some variants of OCT have been developed, including 'high-definition OCT' in which greater optical resolution is obtained with a higher optical power objective lens at the expense of lower image penetration depth and reduced field of view, and 'en-face' OCT in which a 'top-down' view of the tissue at a given depth is viewed.

OCT is a 'passive' imaging technique that does not require a contrast agent. It cannot detect fluorescent markers. Its imaging depth of $0.5-1 \mathrm{~mm}$ is considerably higher than reflectance confocal microscopy (RCM), <0.2 mm [3], but, unlike RCM, it cannot resolve individual cells. The image resolution and contrast of OCT is considerably higher than ultrasound. OCT is ideal for imaging subsurface skin microstructure in the epidermis and upper dermis. In this review, we will use the term 'structural OCT' for all of the above described variants of OCT, because they all detect and image tissue structure.

Structural OCT has been found not to be suitable for diagnosing melanoma, because the images have not shown any features that could enable differentiation between malignant melanomas from dysplastic nevi. However, a novel variation of OCT has recently emerged which shows considerable promise in changing this unsatisfactory situation. This newly developed technique is variously called speckle-variance OCT [4], optical microangiography [5], or dynamic OCT (D-OCT) [6]. It relies on detecting motion in the OCT images: OCT images of the exact same site are captured at a high frame rate and then changes between consecutive OCT image frames are detected, so that moving particles can be distinguished from the static tissue signal. This particle motion corresponds to blood flow, and so D-OCT when coupled with structural OCT reveals both the tissue structure and the morphology of the blood vessel structure in the imaged region. Thus, by using D-OCT to additionally image the blood vessels in skin lesions, it seems feasible that the utility of OCT could be extended to include diagnosis/ prognosis of malignant melanoma and other skin pathologies.

The current review focuses on initial findings using a commercially available, CE-marked D-OCT device, starting with imaging of healthy skin, and moving on to D-OCT images of non-melanoma skin cancer, melanocytic lesions, and then a variety of other conditions chosen by us. For each lesion class, we also briefly review the key features visible in structural OCT images. The work was supported by funds from the European Union (ADVANCE, Automatic Detection of VAscular Networks for Cancer Evaluation, Grant No. 621015). The core aim of the ADVANCE project was to investigate the vasculature in malignant melanomas and correlate the patterns to known risk factors and prognosis.

\section{METHODS}

The device used in the ADVANCE project was a VivoSight Dx multi-beam OCT dermatological scanner (Michelson Diagnostics, Maidstone, UK) equipped with proprietary Dynamic OCT imaging software. The optical resolution of the scanner is $7.5 \mu \mathrm{m}$ in the lateral direction and $5 \mu \mathrm{m}$ in the axial direction. This is sufficient to clearly show fine details of skin morphology and also fine blood vessels, but not high enough to reveal individual cells. The field of view of the probe is $6 \mathrm{~mm} \times 6 \mathrm{~mm}$ and the imaging depth is between 1.0 and $2.0 \mathrm{~mm}$. The handheld probe is equipped with a color camera to aid accurate placement on a lesion and to provide a context picture. No gel or other preparation of the skin is required, the imaging procedure is straightforward and simply requires a steady pair of hands.

The OCT laser wavelength is $1300 \mathrm{~nm}$ (near-IR) at which melanin is transparent, and so the imaging works well in skin of any ethnic type and even on strongly pigmented lesions. One OCT image appears as a vertical slice into 
the skin, $6 \mathrm{~mm}$ wide and up to $2 \mathrm{~mm}$ deep. However, in one 30-s scan, 120 such individual images are captured as a $6 \times 6 \times 2 \mathrm{~mm}$ $(\mathrm{w} \times 1 \times \mathrm{d})$ 'stack' of images, which are then automatically processed to also show the horizontal plane view. The stack can then be evaluated in both horizontal and vertical view simultaneously at any desired depth.

The Dynamic OCT algorithm used in VivoSight is tuned to preferentially detect movement due to blood flow, rather than due to small bulk movement of the probe caused by small involuntary movements of the operator or patient. The software displays the detected areas of motion as a red overlay on the standard grey OCT image, with red brightness corresponding to the strength of the blood flow. The horizontal plane view has been further enhanced by fitting the imaging plane to the skin surface, so that the displayed top-view image comprises pixels at any desired exact depth below the skin surface. This is termed the 'fitted plane' and is useful because skin is far from flat, having wrinkles, bumps and so on, and the vascular plexus tends to follow the surface topography. Thus, Dynamic OCT can reveal the pattern of surface blood vessels and also be used to measure their exact depth, and to provide an indication of the strength of blood flow in the vessels. The Dynamic OCT algorithm works well at depths of up to about $0.5 \mathrm{~mm}$; at greater depths, there is too much interference in the signal by background noise. It is hoped that further developments in image processing will extend the useful depth in the future. This article is based on previously conducted studies and does not involve any new studies of human or animal subjects performed by any of the authors.

\section{RESULTS}

\section{Healthy Skin in Different Locations}

Former studies have already shown that healthy skin has a different appearance in structural OCT depending on the various anatomical locations $[7,8]$. However, the typical structure of the skin, which the vertical sections of OCT images visualize, stays mainly the same. Below the entrance signal, which is responsible for the first thin dark layer in OCT pictures, there is the stratum corneum [7]. This can only be seen in palmoplantar skin and appears as a thick, low-echoic layer, consisting of highly scattering, convoluted sweat gland ducts $[7,8]$. Next, the epidermis is depicted in the OCT image, which appears hypo-reflective compared to the more signal-intense dermis. The dermoepidermal junction (DEJ) stands out as a thin line between the darker epidermis and the lighter dermis. The hyper-reflective dermis also contains parts with lower signal intensity like sebaceous glands, hair follicles or vessels [7].

Turning to D-OCT, Themstrup et al. showed that D-OCT can reliably depict and determine the vasculature and its changes in normal skin due to physiological influences on the blood flow [9]. The information about vasculature is added to the structure of the common OCT image. Due to the distinct body regions and their local external and internal influences, the vascular network appears different. For example, in the en face D-OCT image, the healthy skin of the face shows a broader net of the vessels and also an enlargement of the single vessels compared to other body parts (Fig. 1a). The vessels at the fingertips are organized in the form of a live in vivo fingerprint and can therefore be easily discriminated from other vascular networks in the horizontal display (Fig. 1b). In the en face view, the vasculature of healthy skin on the legs seems to follow a reticular order, while the vessels of normal skin on the arms and the trunk are of comma- and dot-like shapes (Fig. 1c-e). At the nail fold, the vessels are arranged parallel to the skin surface (Fig. 1f). Anatomical variations of the epidermal thickness should also be kept in mind, since the vessels pop up at different levels below the surface in the D-OCT images.

In order to fully understand the vascular network, not only the anatomical but also the physiological influences on vasculature and microcirculation have to be taken into account. The vascular architecture is characterized by two plexuses, which are aligned parallel to the 

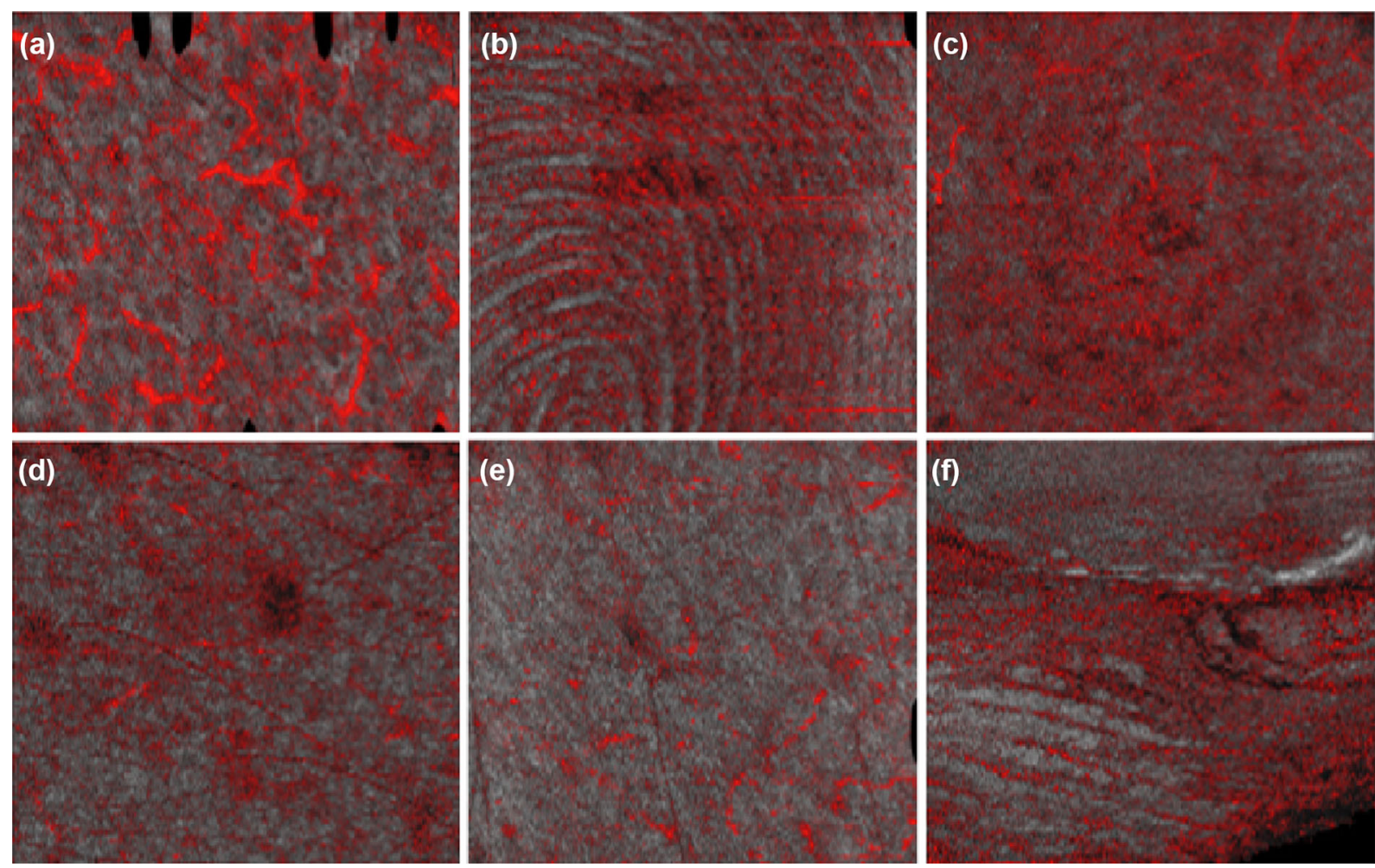

Fig. 1 The skin depth of all depicted images is $0.4 \mathrm{~mm}$. a Normal skin on the left cheek (size: $6 \mathrm{~mm} \times 6 \mathrm{~mm}$, en face view). A broader, but regular vascular network and thicker single vessels in the face in comparison to other body parts can be seen. $\mathbf{b}$ Healthy skin on the fingertip of the left index finger (size: $6 \mathrm{~mm} \times 6 \mathrm{~mm}$, en face view). The vessels are arranged in the form of a live in vivo fingerprint. c-e Normal skin on the right leg, left arm and

surface of the skin. The superficial plexus between the papillary and reticular dermis is the source of the capillary loops for the papillae, which nurture the avascular epidermis [10]. These loops demarcate as small dots along the DEJ in vertical D-OCT images (Fig. 2). The profound plexus between the dermis and subcutis connects with the superficial plexus through vertical vessels in a healthy state.

In general, normal skin can be detected in D-OCT based on a well-arranged vascular pattern and each of the vessels is clearly visible in the en face images. These two characteristics need to be considered in order to distinguish the vasculature of healthy skin from diseased skin. trunk (size: $6 \mathrm{~mm} \times 6 \mathrm{~mm}$, en face view). The vasculature of healthy skin on the legs is organized in a reticular pattern, the vessels of normal skin on the arms and the trunk come in form of commas and dots. f Normal skin on the left nail fold of the left index finger (size: $6 \mathrm{~mm} \times 6 \mathrm{~mm}$, en face view). At the nail fold, the vessels are arranged parallel to the skin surface

\section{Skin Tumors}

\section{Actinic Keratosis, Bowen's Disease, Invasive Squamous Cell Carcinoma}

In structural OCT images, actinic keratoses (AK) show a thickening of the stratum corneum and of the epidermis. All layers can be discriminated from each other and the DEJ is demarcated as a dark line between the epidermis and dermis. There are varying degrees of hyperkeratosis, for example the highly reflecting forms which are often found in thick hyperkeratotic AK, but also variants of hypo-reflection due to parakeratosis. D-OCT depicts the vasculature of AK in the en face view as a reticular pattern with more and slightly larger vessels and in greater disarray 


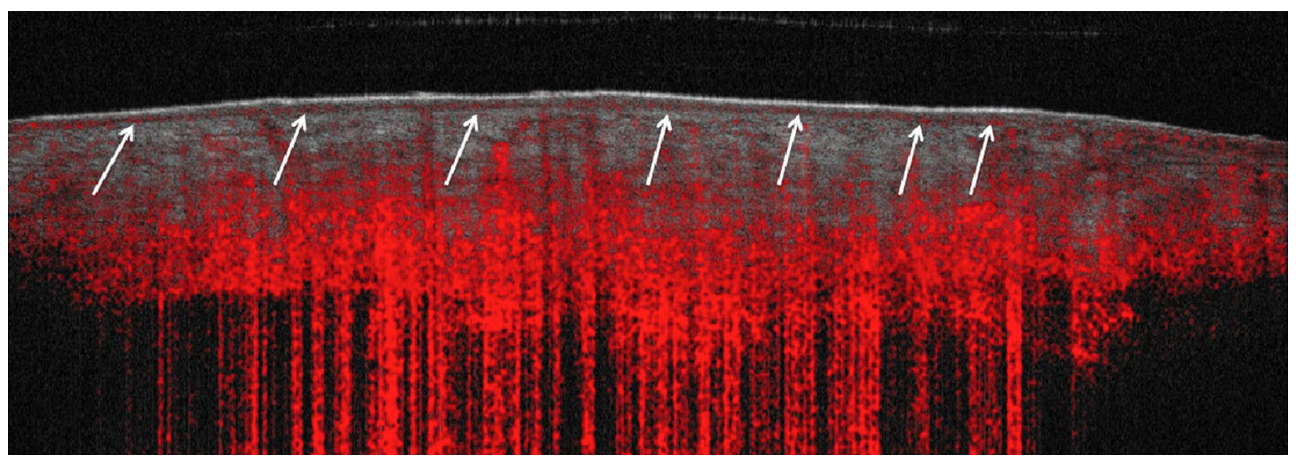

Fig. 2 Normal skin on the right leg (size: $6 \mathrm{~mm} \times 2 \mathrm{~mm}$, vertical view). The capillary loops for the papillae, which supply the avascular epidermis and arise from the superficial plexus, are shown as small dots along the DEJ (arrows) in vertical D-OCT images. The vertical stripes at the base of the image are artifacts of the processing algorithm and can be ignored
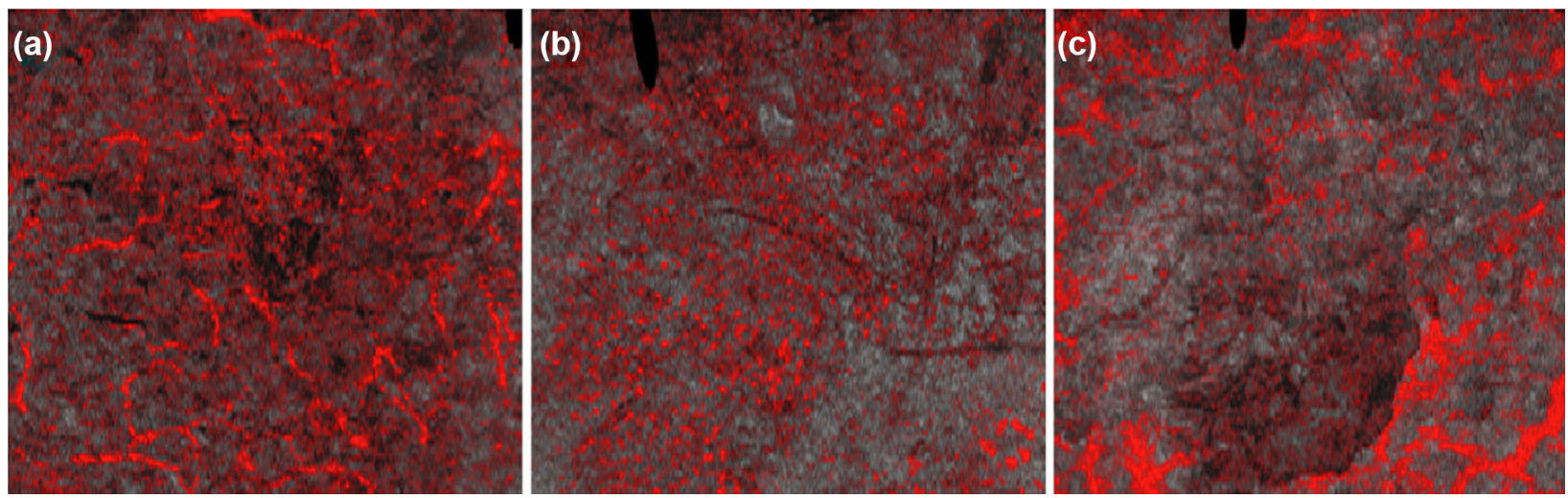

Fig. 3 a AK on the forehead (size: $6 \mathrm{~mm} \times 6 \mathrm{~mm}$, en face view). The skin depth of the depicted image is $0.3 \mathrm{~mm}$. The vessels in AK are arranged in a reticular network with more and slightly bigger vessels in contrast to normal skin. b Bowen's disease/SCC in situ on the left hand (size: $6 \mathrm{~mm} \times 6 \mathrm{~mm}$, en face view). The skin depth of the depicted image is $0.2 \mathrm{~mm}$. The vasculature of Bowen's

compared to healthy skin (Fig. 3a). Thick and thin branching vessels are present [11].

In conventional OCT, Bowen's disease/ squamous cell carcinoma (SCC) in situ have a lot in common with $\mathrm{AK}$, since there is, for example, also a visible increase in epidermal thickness. Typical features like hyperkeratotic scales appear less thick or are even absent. But with D-OCT, it might be possible to differentiate between the two skin tumor entities by the vasculature. The vascular network in Bowen's disease/SCC in situ has a more grainy
disease/SCC in situ mainly consists of dots, blobs and less curved vessels and is not organized in a network. $\mathbf{c}$ Invasive SCC on the left cheek (size: $6 \mathrm{~mm} \times 6 \mathrm{~mm}$, en face view). The skin depth of the depicted image is $0.4 \mathrm{~mm}$. The vascular network of invasive SCC is of irregular morphology with diffusely organized vessels in changing diameters

appearance with vessels in the form of dots, blobs and sometimes curves, dependent on the skin depth of the image (Fig. 3b). Overall, the dot-like vessels in Bowen's disease/SCC in situ are larger than in healthy or psoriatic skin.

Invasive SCC, in situ SCC and AK with thick hyperkeratotic scales are very difficult to discriminate with structural OCT images mainly because the hyperkeratosis makes it nearly impossible to get an insight into deeper skin layers and the DEJ is also obscured. In the structural OCT images, the diagnostician has to 
identify specific characteristics of invasive SCC such as the alteration or loss of the normally visible DEJ as well as the disruption of the dermis and/or of regular skin layers, which can also be noticed in AK/SCC in situ. In the dermis, protrusions from the epidermis invading the dermis show various shapes of different signal intensity. With D-OCT, the dynamic mode adds information on the vasculature to the structural images. The vessels of invasive SCC appear in different irregular forms and patterns on the one hand and show changes in vessel diameters on the other (Fig. 3c).

\section{Basal Cell Carcinoma}

$\mathrm{BCC}$ is the most common skin cancer and also the most frequent non-melanocytic skin cancer. The most important risk factor for BCC is UV irradiation [12]. Initially, the BCC can be clinically detected as small, highly demarcated, translucent or pearly nodules, papules, scars or ulcerations with typical teleangiectasia $[12,13]$. There are different subtypes of BCC with associated risks of recurrences [14]. BCC grow slowly, but, in the absence of treatment, the tumor can expand in the vertical as well as the horizontal direction and lead to destruction of tissue or even bones [13]. The rate of metastasis is very low and thus BCCs are sometimes called semi-malignant tumors [12].
In structural OCT, a BCC is apparent as an ovoid hypo-reflective formation with a darker boundary and partially signal-free, cystic parts compared to the surrounding hyper-reflective stroma, or is visible as a lobule-like protrusion of the epidermis. An alteration of the DEJ is also a typical feature of BCC in OCT. Clinically, a BCC can be diagnosed with a sensitivity of $59-90 \%$ and a specificity of $75-90 \%$, while with dermoscopy and OCT a precision of $87.4 \%$ can be achieved $[2,15]$. Nevertheless, it is still difficult to discriminate between BCC subtypes despite a new proposed algorithm in high-definition OCT [16-19]. As well as for initial diagnosis, OCT is also used for treatment monitoring of BCC [20-23].

As for every type of tumor, the extension and growth of vessels are essential for the development of non-melanoma skin cancer [24]. Due to angiogenesis, the necessary nutrients and oxygen reach the BCC and help to support its growth. In BCC, there are two known forms of blood vessels. The superficial arborizing vessels, named teleangiectasia, can be recognized in dermoscopy, while in the histopathological examination multifocal superficial BCC are displayed by light tissue and a higher vascularisation in the dermis between the multiple tumor islands [25]. Both the superficial and the deeper vascular networks in the dermis can be

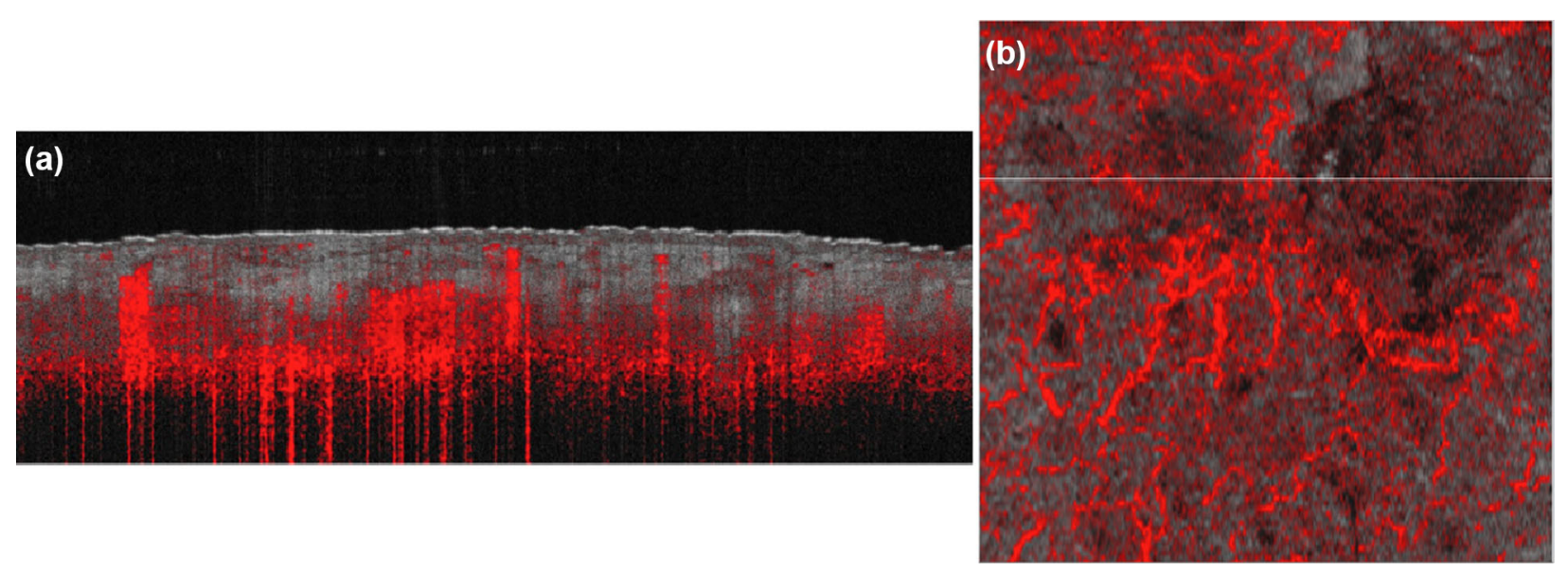

Fig. 4 a BCC on the back (size: $6 \mathrm{~mm} \times 2 \mathrm{~mm}$, cross-sectional view). Extended vertical vessel columns can be seen in superficial BCCs. b BCC on the nose (size: $6 \mathrm{~mm} \times 6 \mathrm{~mm}$, en face view). The skin depth of the depicted image is $0.3 \mathrm{~mm}$. The vessels in BCC show changes in diameter and are distributed disorderly. The broad pattern of the vasculature is located in the center and around the edges of the tumor nests 
seen in the D-OCT images. First, the cross-sectional D-OCT images show extended vertical vessel columns in superficial BCC (Fig. 4a). Second, the en face view visualizes the changes in vessel diameters and the chaotic arrangement of very small irregular vessels (Fig. 4b) [11]. There is a wide range of the vascular network in BCC visible. Some vessels appear larger than those seen in healthy skin and some look so tiny and fragile that they would not have been noticed if they were not depicted in red color. It is obvious that the vessels are mainly located in the center of the BCC, and sometimes it can be seen that the vessels also seem to surround the rims of the tumor nests (Fig. 4b) [6]. The additional information regarding the vessel morphology in BCCs provided by D-OCT may be of value in non-invasively determining the subtype and aggressiveness of the tumour, so that more informed treatment decisions can be made, especially in the context of non-invasive treatment options.

\section{Melanocytic Nevus and Malignant Melanoma}

Due to the limitation of the image resolution, it is difficult to evaluate melanocytic lesions in conventional cross-sectional OCT images. However, with D-OCT, it is necessary to observe the vasculature of nevi or melanoma in horizontal or even $3 \mathrm{D}$ views.
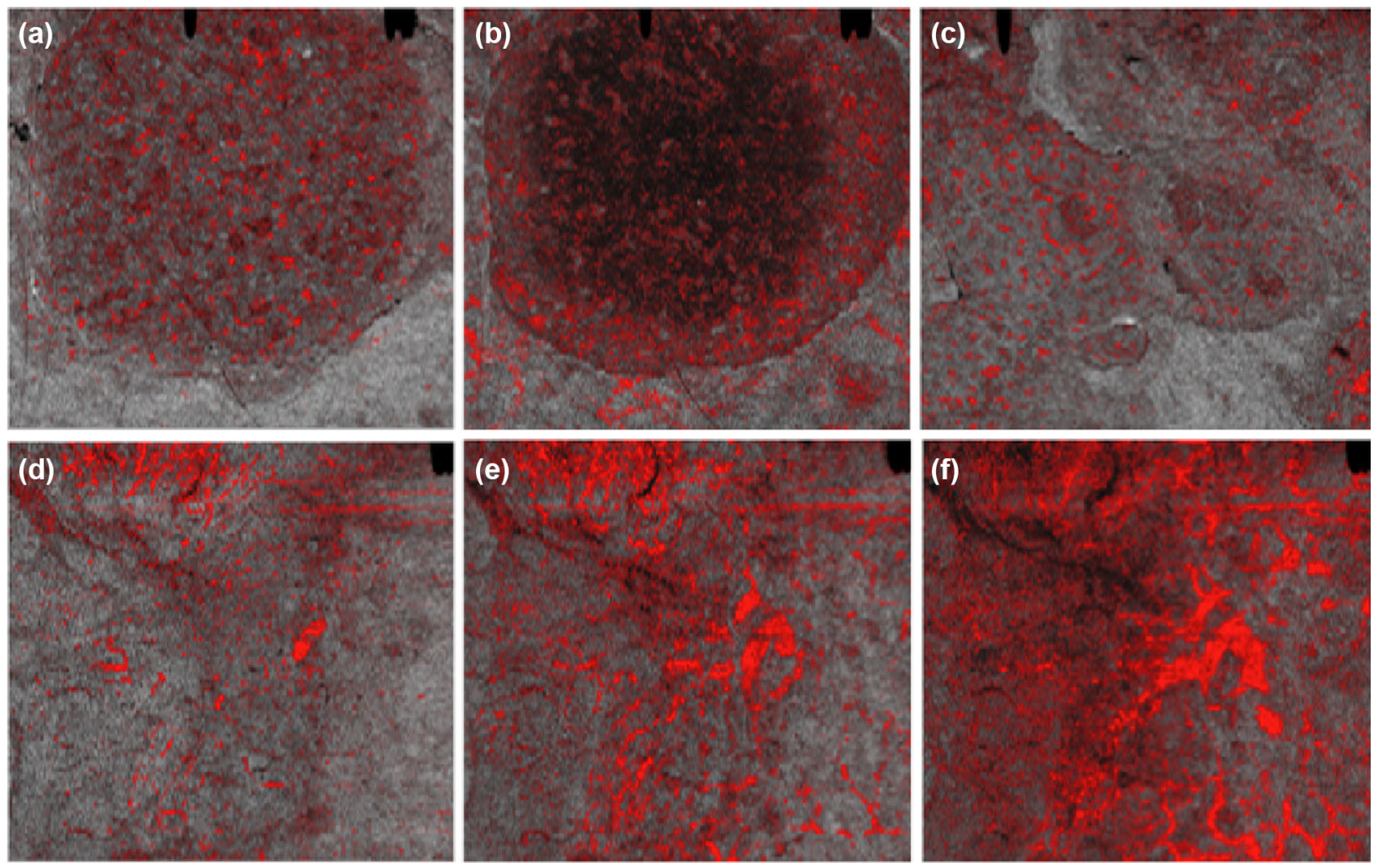

Fig. 5 a, b A dermal nevus on the right abdomen (size: $6 \mathrm{~mm} \times 6 \mathrm{~mm}$, en face (superficial and deeper) view). The skin depth of the depicted image is 0.2 and $0.4 \mathrm{~mm}$. Under the epidermis inside the mole, dotted vessels are regularly distributed. If there is a deep dermal involvement then sometimes a precise reticular pattern is visible. c A seborrheic keratosis on the left breast (size: $6 \mathrm{~mm} \times 6 \mathrm{~mm}$, en face view). The skin depth of the depicted image is $0.4 \mathrm{~mm}$. Many regularly distributed dotted, curved, and also coiled vessels are visible. d-f A malignant melanoma on the left shoulder (size: $6 \mathrm{~mm} \times 6 \mathrm{~mm}$, en face view). The skin depths of the depicted image are $0.2,0.3$ and $0.4 \mathrm{~mm}$. Clusters of vessel dots and even blobs in the superficial dermis are diffusely distributed. With increase in the tumor depth, the dots seem to build linear formations. This invasive melanoma shows long linear vessels of irregular size and distribution with a branching architecture 
In nevi with accumulations of melanocytes in the DEJ or in the superficial dermis, the D-OCT shows a homogenous picture like in healthy adjacent skin. Right below the epidermis inside the mole, sharply demarcated vessels regularly organized in the form of dots are visible (Fig. 5a). If there is a deep dermal involvement, then sometimes a delicate reticular pattern can be noticed (Fig. 5b). In a suspected dysplastic lesion, the vessels in the dermis are larger and formed in characteristic curves, reaching from bottom to top with regular frequency. To visualize this aspect, a 3D image must be constructed from the D-OCT dataset. The tumor depth is directly proportional to the length of the bowed vessels.

Because of the intra-epidermal growth of melanomas, the tumors show scattered distributed clusters of vessel dots and even blobs in the superficial dermis (Fig. 5d) [26]. However, the deeper the tumor grows, the more that dots seem to form linear figures (Fig. 5e). Highly invasive melanomas consist of long, intense, linear vessels that are of irregular size and distribution and show a branching pattern (Fig. 5f). The latter is visualized as an arborizing or bulging architecture. Especially in thick, deeply invasive melanomas, the formation of vessel aneurysms and multiple bizarrely and irregularly shaped vessels are apparent. In 3D view, it can be seen that these tumors build 'spikes', delicate perpendicular figures, which reach up to the epidermal surface.

Our preliminary results indicate that D-OCT reveals changes in vessel morphology as the lesion progresses from dysplastic nevus to melanoma in situ and then to more invasive melanomas. We therefore speculate that D-OCT might be useful in assessing disease progression leading to earlier/more efficient interventions, and also as a powerful research tool in understanding this disease. Further work is required to confirm these ideas.

\section{Seborrheic Keratosis}

In structural OCT in the cross-sectional as well as in the en face views, the pattern of gyri and sulci is one typical criterion of seborrheic keratoses. However, when looking at the vessels, the benign character of the lesion is immediately obvious. There are many regularly distributed dotted, but also curved, and sometimes even coiled vessels corresponding to the hairpin-like vessels, which can also be found in dermoscopy (Fig. 5c).

\section{Inflammatory and Degenerative Skin Diseases}

Skin diseases, which are caused due to inflammation or degeneration, can show changes in the size, amount, diameter and
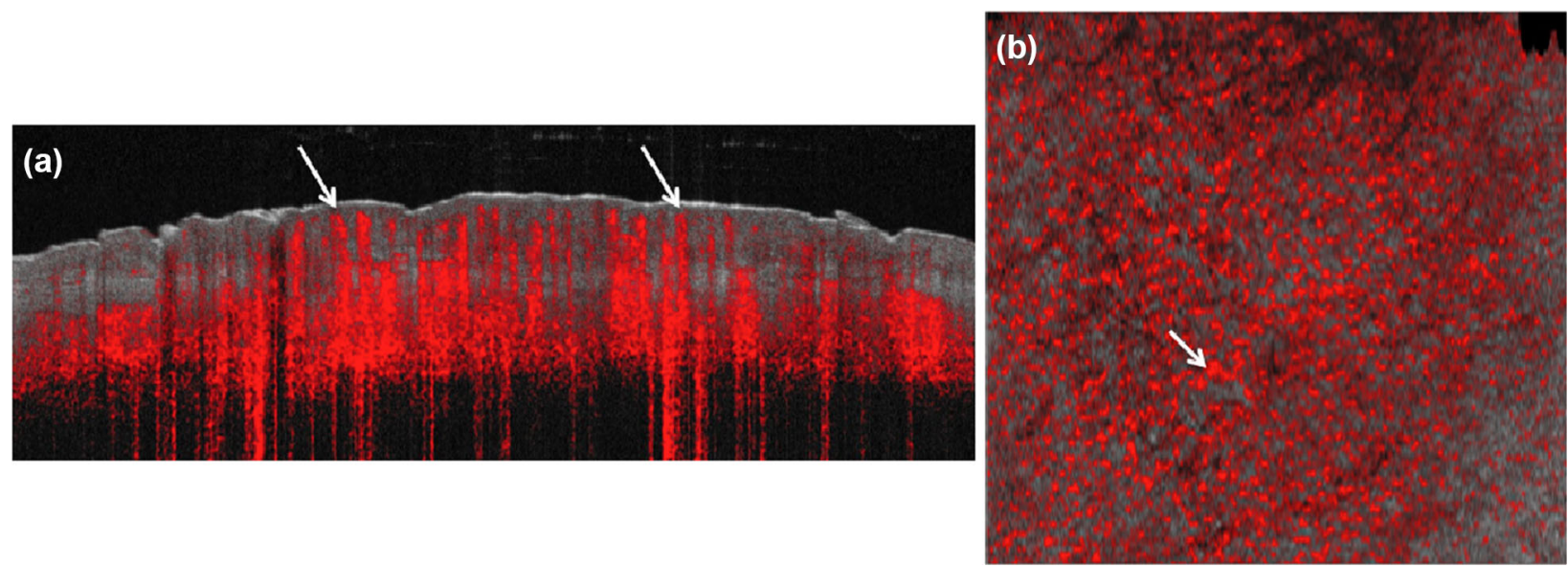

Fig. 6 Psoriasis on the left elbow with a thickening of the stratum corneum and the epidermis as well as visible hypervascularisation: a size: $6 \mathrm{~mm} \times 2 \mathrm{~mm}$, cross-sectional view; b size: $6 \mathrm{~mm} \times 6 \mathrm{~mm}$, en face view. The skin depth in en face view is $0.3 \mathrm{~mm}$. In the papillary dermis, the extended vessel loops are apparent (arrows) 
three-dimensional structure of their blood vessels. For example, erythematous skin arises because of the vascular dilatation. In this context, D-OCT may be of value in non-invasively monitoring and measuring the effects of treatments.

\section{Psoriasis}

Psoriatic skin shows hyperkeratosis, acanthosis, pronounced regular rete ridges and dilated vessels in the dermis, demonstrating the components of inflammation, proliferation of the epidermis and angiogenesis, which contribute to the development of psoriasis. All of these features are displayed in structural OCT, but, especially with D-OCT, the typical vessel capillary loops in the papillary dermis, named spikes, are visible (Fig. 6a, b).

\section{Scleroderma}

In patients with systemic scleroderma, it is known that this disease causes thickening and hardening of the skin and impedes the microcirculation blood supply especially in the fingertips [27-29]. Ring et al. found out that in structural OCT the typical DEJ is missing and the density of the dermis is enhanced along with a disappearance of the skin appendages [30]. As a result of the loss of blood vessels, the acral parts of the body like the fingertips are no longer supplied with blood leading to necrosis and ulceration. A recent study discovered
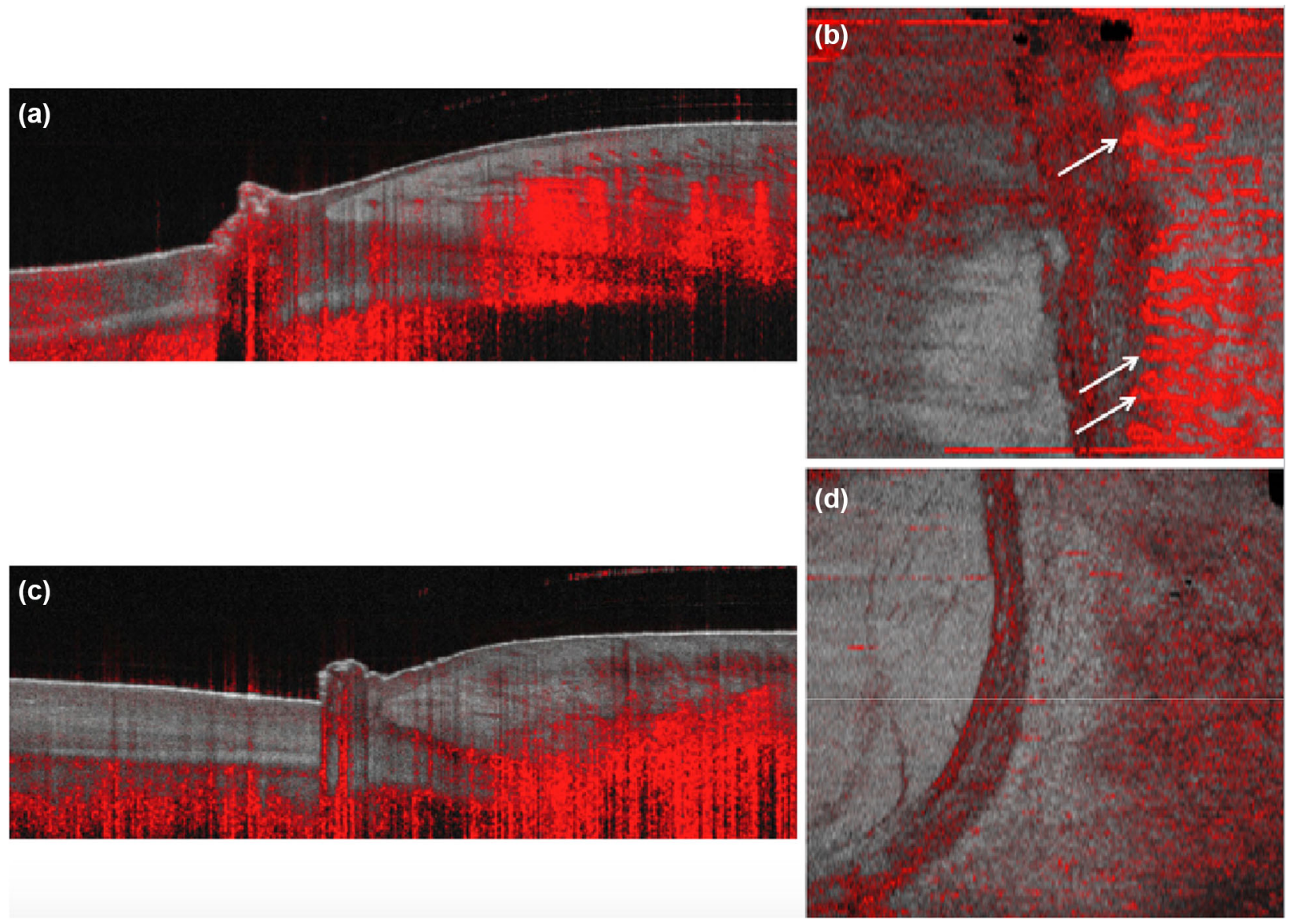

Fig. 7 Comparison of a nail fold in a scleroderma patient (a, b) with a normal nail fold in a healthy patient (c, d) (size: $6 \mathrm{~mm} \times 2 \mathrm{~mm}$, cross-sectional view, size: $6 \mathrm{~mm} \times 6$ $\mathrm{mm}$, en face view). A thickening of the epidermis and dermis and a loss of the vasculature can be noticed in scleroderma compared to normal skin. Despite that, megacapillaries in the shape of a Y (white arrows) are found in scleroderma, which take the place of the compact network of small vessels seen in healthy skin 
megacapillaries in the shape of a Y with D-OCT instead of the small vascular network, which can be recognized in healthy nail folds $[6,30]$ [Fig. 7 comparison of a scleroderma patient (a, b) with a healthy skin fold (c, d)].

\section{Wounds}

The distinction between different types of chronic wounds is often difficult. A biopsy will probably lead to the final diagnosis, but is counterproductive for delayed wound healing. To investigate the healing process, biopsy is not
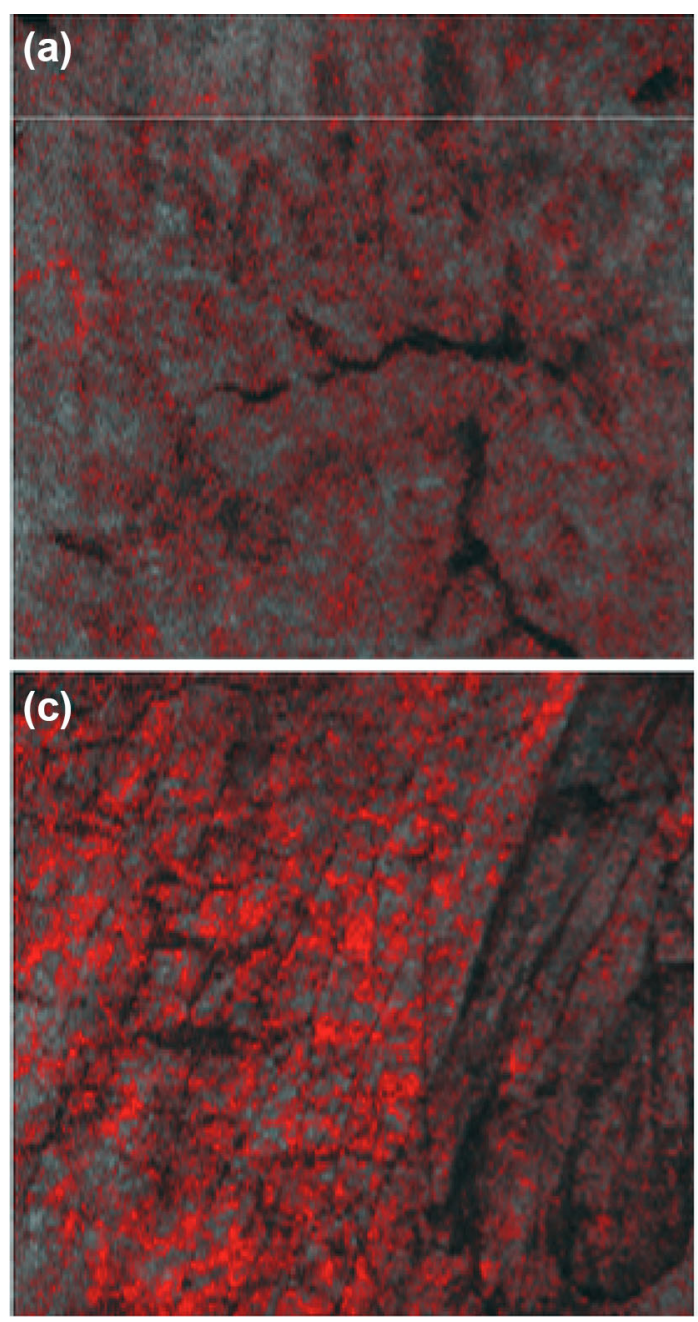

Fig. 8 Patient with a chronic venous ulcer on the lower extremity (size: $6 \mathrm{~mm} \times 2 \mathrm{~mm}$, cross-sectional view, size: $6 \mathrm{~mm} \times 6 \mathrm{~mm}$, en face view). a Adjacent normal skin in the surrounding area of the ulcer with extended lymphatic vessels without perfusion. b Dermatosclerotic skin with the ideal way to do this, because, once the tissue sample is taken, repeated biopsies are not possible in the same location. Through the use of D-OCT, in vivo insight into the wound with its newly proliferating vessels enables multiple non-invasive "biopsies" from several locations which may provide full monitoring of the continuous wound healing process like no other available device [31, 32].

In particular in chronic venous insufficiency, different stages of vascularisation are visible. The adjacent normal skin in the surrounding
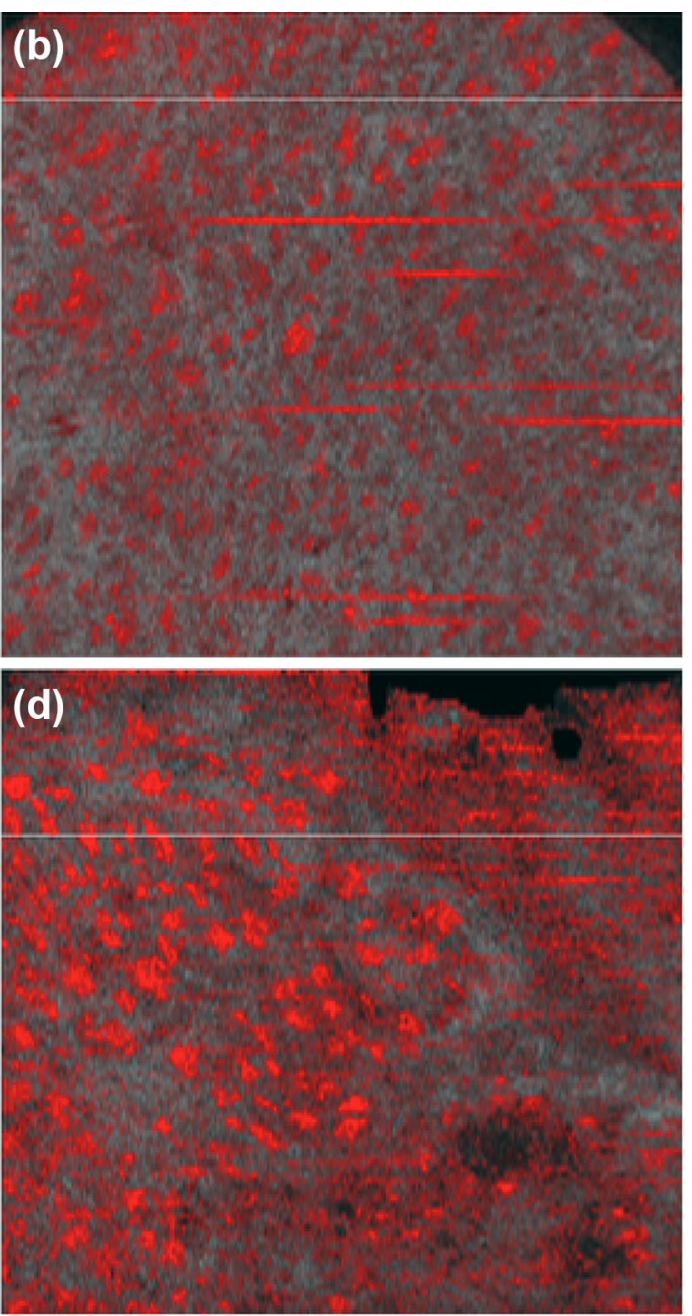

minimal increase of dotted vessels. $\mathbf{c}$ The margin of the ulcer shows larger vessel dots of higher density. $\mathbf{d}$ In the middle of the ulcer, larger crowds of dotted vessels, called blobs, due to granulation are visible 

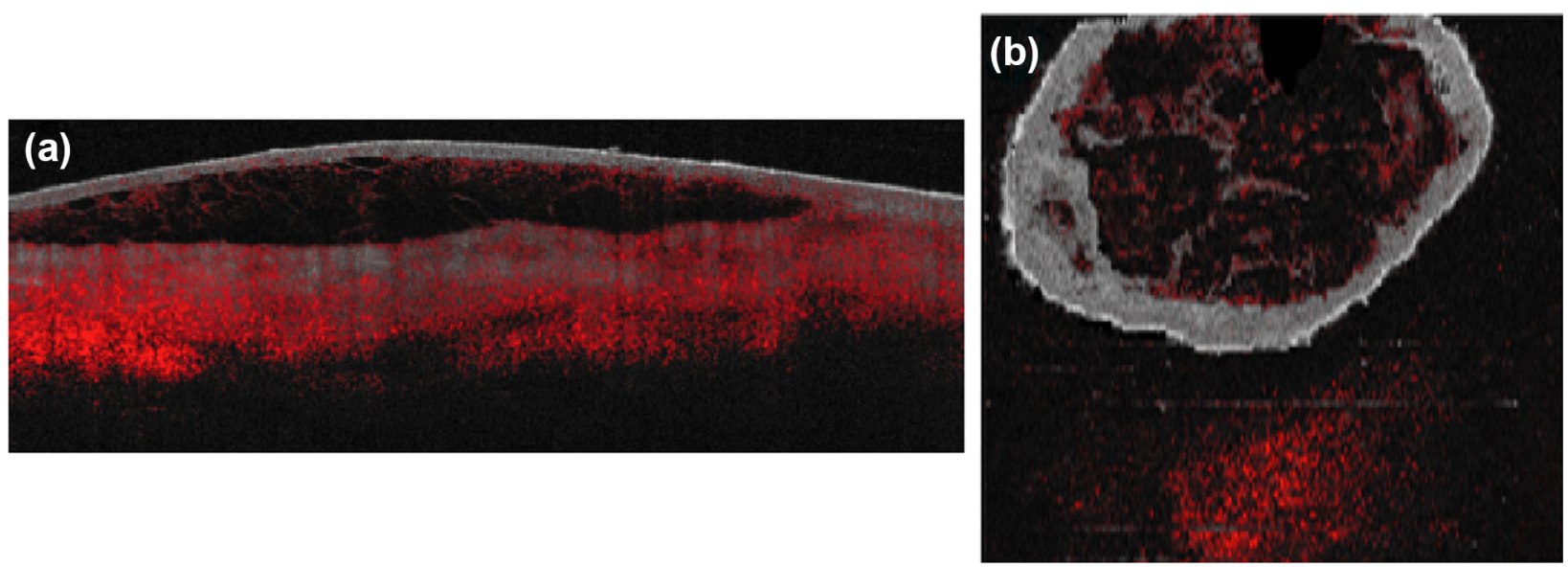

Fig. 9 Burn injury grade IIa with a subepidermal blister on the knee: a size: $6 \mathrm{~mm} \times 2 \mathrm{~mm}$, cross-sectional view; b size: $6 \mathrm{~mm} \times 6 \mathrm{~mm}$, en face view. Below the bulla, the presence of active blood vessels indicates a burn wound grade IIa

area of the ulcer presents large, extended lymphatic vessels without perfusion (Fig. 8a). In dermatosclerotic skin, there is only a minor growth of dotted vessels in the sclerotic and fibrous stroma (Fig. 8b). At the margin of the ulcer, the vessels form dots of higher density (Fig. 8c). In the middle of the wound itself, granulation islands with larger aggregations of vessels in the form of dots, blobs and coils are apparent (Fig. 8d). It might therefore be possible to use D-OCT to assess the healing status of individual ulcers so that 'chronic' non-healing ulcers can be quickly identified for earlier intervention than is currently possible.

Moreover, D-OCT may help to evaluate the depth of a burn wound and to determine whether the injury can be treated conservatively or surgically [33-36]. Besides the structural information on the amount of burned skin, D-OCT shows the remaining and the thermolytically affected blood vessels (Fig. 9a, b). This way, the diagnostician may get an overall impression of the extent of the burn injury by using D-OCT.

\section{Scars}

Scars are the end result of tissue repair. Thus, there is still a difference between healthy skin and scar tissue. Clinically scars can be painful or itchy; they can be atrophic, hypertrophic or even keloids, which exceed the original scar area with their proliferation. Besides that, scars are distinguishable from normal skin through their color/pigmentation, structure, height in the level of the skin, firmness and pliability. Histopathologically, alterations in the vascular network and in the epidermal structure can be found in scar tissue, but collagen deposits, fibrous tissue remodeling, inflammatory infiltrations and sclerosis might also be present.

All these criteria need to be considered for the evaluation of scars. Nevertheless, only

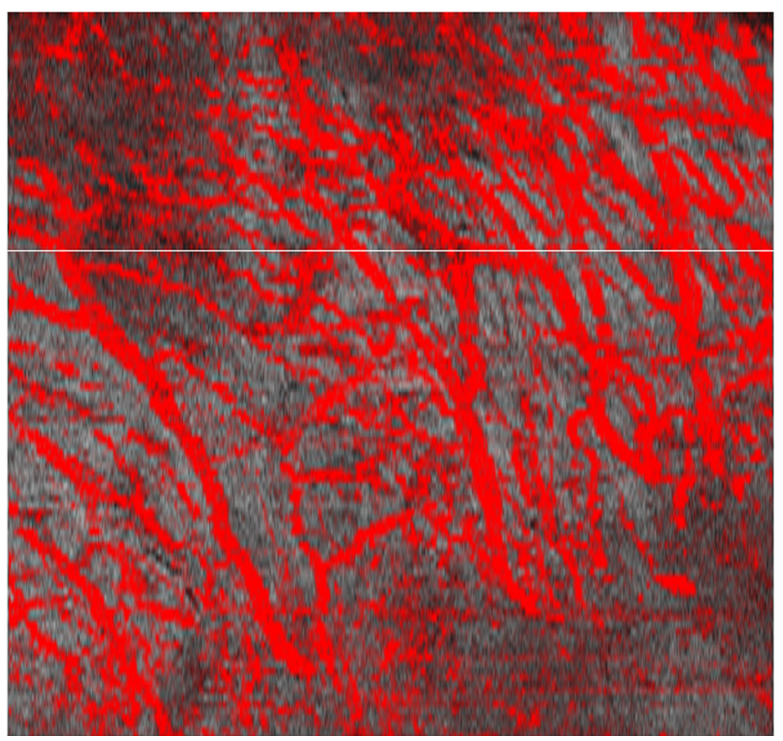

Fig. 10 Scar on the right temple (size: $6 \mathrm{~mm} \times 6 \mathrm{~mm}$, en face view). Scars show a lot more vessels and in a streaming pattern, which means the blood vessels are aligned parallel to each other 

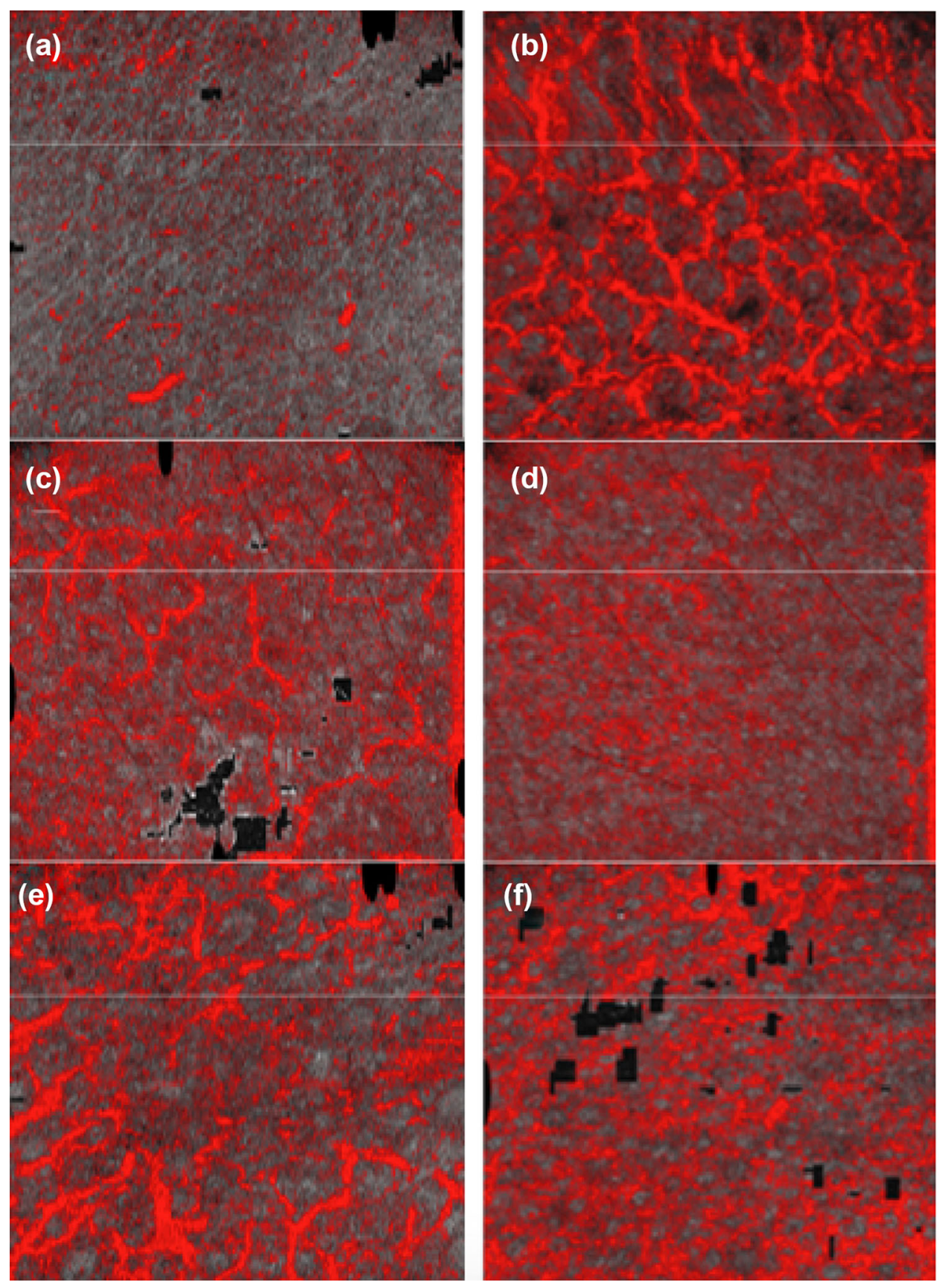

Fig. 11 a, b Cheek of a rosacea patient (size: $6 \mathrm{~mm} \times 6 \mathrm{~mm}$, en face view). The skin depths in en face view are $0.2 \mathrm{~mm}$ and $0.4 \mathrm{~mm}$. a Small dotted vessels in superficial skin layers represent the erythema. b The broad vascular network visualizes the teleangiectasia (a, b). c, d Cheek of a healthy person (size: $6 \mathrm{~mm} \times 6 \mathrm{~mm}$, en face view). The skin depth in en face view is $0.4 \mathrm{~mm}$.

Superficial blood vessels before (c) and after (d) the application of topical brimonidine. After treatment, a vascular constriction is visible (d). e, $\mathbf{f}$ Cheek of a rosacea patient (size: $6 \mathrm{~mm} \times 6 \mathrm{~mm}$, en face view). The skin depth in en face view is $0.4 \mathrm{~mm}$. The former broad vascular network (e) is replaced by a fuzzy appearance after dye laser therapy $(\mathbf{f})$ 
subjective scores are available and objective parameters are missing $[37,38]$. Moreover, scars after burn injury were objectively explored with OCT by Gong et al. [37, 39] and Liew et al. [40] through automated quantification of the density and diameter of the vasculature using speckle decorrelation and the attenuation coefficient $[37,39,40]$. The result is that inside the scar tissue the density of blood vessels is increased and that there is no typical vascular network formation like in healthy skin, but a parallel organization of the vasculature (Fig. 10). More effective characterization of scars with D-OCT may lead to more accurate treatments.

\section{Evaluation of Treatment Effects}

D-OCT is also a useful device for the visualization and evaluation of treatment effects. Rosacea patients often show erythema and teleangiectasia, because of the dilatation of thin superficial blood vessels. Erythema is visualized in D-OCT as small dotted vessels in superficial skin layers and, in deeper levels, the teleangiectasia show a network of vessels with a broader diameter than in healthy skin (Fig. 11a, b). There are treatment options to reduce these alterations by the mechanism of vasoconstriction. With D-OCT, it is possible to observe the superficial blood vessel changes in erythemato-teleangiectatic rosacea, and also in normal skin, which were locally treated with brimonidine gel $0.33 \%$ [41, 42] (Fig. 11c, d). This is a selective $\mathrm{a}_{2}$-adrenergic receptor agonist, which peripherally constricts the superficial vessels [41, 42].

In rosacea, both the superficial and the deeper vascular network are affected by the disease. Since there is a limitation in the penetration for the topical treatment with brimonidine, dye laser therapy is used for the coagulation of deeper blood vessels. In D-OCT, the former broad vascular network is replaced by a fuzzy appearance of the treated vasculature (Fig. 11e, f). Ring et al. proved that, through intense pulsed light therapy, the microvascular destruction can be imaged even by structural
OCT [43]. Using D-OCT, these vascular changes can be quantitatively evaluated before and after topical brimonidine as well as laser treatment.

\section{CONCLUSIONS}

Dynamic OCT is a new and powerful unique tool of investigating in vivo blood vessels of superficial skin layers. The pattern of perfusion plays an important role not only in inflammatory skin diseases but also in skin tumors. Neo-angiogenesis is a typical feature of neoplastic tumors, and metastases occur via lymphatic and blood vessels. Our studies on actinic keratoses, squamous cell carcinomas, Bowen's disease and basal cell carcinomas exhibited different patterns of vasculature between the entities as well as changes corresponding to tumor invasion. In melanomas, an increased vasculature with atypical branching was found. Further studies are necessary to correlate the degree of vessel atypia with the risk of metastasis. If this is proven, D-OCT might enable earlier and more accurate treatment for improved patient outcomes.

Other interesting fields for D-OCT are wound healing and pharmacologic effects. We could demonstrate that pharmacologic effects on perfusion can be easily quantified and monitored over time using D-OCT. Furthermore, inflammatory and degenerative skin diseases as well as acute and chronic wounds show characteristic changes of the blood vessels, allowing for quantification and monitoring of these diseases in a non-invasive way.

\section{ACKNOWLEDGEMENTS}

This work is the result of an EU-funded project ADVANCE, Project Number 621015. All named authors meet the International Committee of Medical Journal Editors (ICMJE) criteria for authorship for this manuscript, take responsibility for the integrity of the work as a whole, and have given final approval for the version to be published. 
Disclosures. Martina Ulrich is a stakeholder in CMB Collegium Medicum Berlin GmbH and has participated in clinical trials sponsored by Almirall, Biofrontera, Leo Pharma and Novartis; she has served as a paid speaker for Almirall, Biofrontera, Galderma, Leo Pharma, Mavig $\mathrm{GmbH}$ and Michelson Diagnostics. Jon Holmes is an employee of Michelson Diagnostics. Gregor B. E. Jemec reports receiving fees for serving on advisory boards from AbbVie, Merck Sharp \& Dohme, and Pfizer, lecture fees from AbbVie, Merck Sharp \& Dohme, LEO Pharma, and Galderma, and grant support from AbbVie, Actelion, Janssen-Cilag, LEO Pharma, Novartis, and Regeneron. Sandra Schuh, Lotte Themstrup, Nathalie De Carvalho, Giovanni Pellacani and Julia Welzel have nothing to disclose.

Compliance with Ethics Guidelines. This article is based on previously conducted studies and does not involve any new studies of human or animal subjects performed by any of the authors.

Open Access. This article is distributed under the terms of the Creative Commons Attribution-NonCommercial 4.0 International License (http://creativecommons.org/licenses/ by-nc/4.0/), which permits any noncommercial use, distribution, and reproduction in any medium, provided you give appropriate credit to the original author(s) and the source, provide a link to the Creative Commons license, and indicate if changes were made.

\section{REFERENCES}

1. Welzel J, Lankenau E, Birngruber R, Engelhardt R. Optical coherence tomography of the human skin. J Am Acad Dermatol. 1997;37(6):958-63.

2. Ulrich M, von Braunmuehl T, Kurzen H, Dirschka T, Kellner C, Sattler EC, et al. The sensitivity and specificity of optical coherence tomography for the assisted diagnosis of nonpigmented basal cell carcinoma: an observational study. Br J Dermatol. 2015;173(2):428-35.

3. Longo C, Pellacani G. Reflectance confocal microscopy. In: Katsambas AD, Lotti TM, Dessinioti C, D'Erme AM, editors. European handbook of dermatological treatments. Berlin:Springer;2015. p. 1129-37.

4. Mariampillai A, Standish BA, Moriyama EH, Khurana $M$, Munce NR, Leung MK, et al. Speckle variance detection of microvasculature using swept-source optical coherence tomography. Opt Lett. 2008;33(13):1530-2.

5. Wang RK, An L, Saunders S, Wilson DJ. Optical microangiography provides depth-resolved images of directional ocular blood perfusion in posterior eye segment. J Biomed Opt. 2010;15(2):020502.

6. Ulrich M, Themstrup L, de Carvalho N, Manfredi M, Grana C, Ciardo S, et al. Dynamic optical coherence tomography in dermatology. Dermatology. 2016;232(3):298-311.

7. Welzel J. Optical coherence tomography in dermatology: a review. Skin Res Technol. 2001;7(1):1-9.

8. Mogensen M, Morsy HA, Thrane L, Jemec GBE. Morphology and epidermal thickness of normal skin imaged by optical coherence tomography. Dermatology. 2008;217(1):14-20.

9. Themstrup L, Welzel J, Ciardo S, Kaestle R, Ulrich $\mathrm{M}$, Holmes J, et al. Validation of dynamic optical coherence tomography for non-invasive, in vivo microcirculation imaging of the skin. Microvasc Res. 2016;107:97-105.

10. Braverman IM. The cutaneous microcirculation. J Invest Dermatol Symp Proc. 2000;5(1):3-9.

11. Markowitz O, Schwartz M, Minhas S, Siegel DM. Speckle-variance optical coherence tomography: a novel approach to skin cancer characterization using vascular patterns. Dermatol Online J. 2016;22(4):1-6.

12. Reifenberger J. Basalzellkarzinom. In: Plewig G, Braun-Falco O, editors. Braun-Falco's Dermatologie, Venerologie und Allergologie. 6th ed. Berlin: Springer; 2012. p. 1633-44.

13. Wong CSM, Strange RC, Lear JT. Basal cell carcinoma. Brit Med J. 2003;327(7418):794-8.

14. Strutton GM. Pathological variants of basal cell carcinoma. Australas J Dermatol. 1997;38(S1):S31-5.

15. Mogensen M, Jemec GBE. Diagnosis of nonmelanoma skin cancer/keratinocyte carcinoma: a review of diagnostic accuracy of nonmelanoma skin cancer diagnostic tests and technologies. Dermatol Surg. 2007;33(10):1158-74.

16. Maier T, Braun-Falco M, Hinz T, Schmid-Wendtner $\mathrm{M}-\mathrm{H}$, Ruzicka T, Berking C. Morphology of basal 
cell carcinoma in high definition optical coherence tomography: en-face and slice imaging mode, and comparison with histology. J Eur Acad Dermatol Venereol. 2013;27(1):e97-104.

17. Boone MALM, Norrenberg S, Jemec GBE, Del Marmol V. Imaging of basal cell carcinoma by high-definition optical coherence tomography: histomorphological correlation. A pilot study. $\mathrm{Br} \mathrm{J}$ Dermatol. 2012;167(4):856-64.

18. Boone MALM, Suppa M, Pellacani G, Marneffe A, Miyamoto M, Alarcón I, et al. High-definition optical coherence tomography algorithm for discrimination of basal cell carcinoma from clinical BCC imitators and differentiation between common subtypes. J Eur Acad Dermatol Venereol. 2015;29(9):1771-80.

19. Gambichler T, Plura I, Kampilafkos P, Valavanis K, Sand M, Bechara FG, et al. Histopathological correlates of basal cell carcinoma in the slice and en face imaging modes of high-definition optical coherence tomography. $\mathrm{Br} \mathrm{J}$ Dermatol. 2014;170(6):1358-61.

20. Banzhaf CA, Themstrup L, Ring HC, Mogensen M, Jemec GBE. Optical coherence tomography imaging of non-melanoma skin cancer undergoing imiquimod therapy. Skin Res Technol. 2014;20(2):170-6.

21. Themstrup L, Banzhaf CA, Mogensen M, Jemec GBE. Optical coherence tomography imaging of non-melanoma skin cancer undergoing photodynamic therapy reveals subclinical residual lesions. Photodiagn Photodyn Ther. 2014;11(1):7-12.

22. Mogensen M, Thrane L, Jørgensen TM, Andersen PE, Jemec GBE. Optical coherence tomography for imaging of skin and skin diseases. Semin Cutan Med Surg. 2009;28(3):196-202.

23. Maier T, Kulichova D, Ruzicka T, Berking C. Noninvasive monitoring of basal cell carcinomas treated with systemic hedgehog inhibitors: pseudocysts as a sign of tumor regression. J Am Acad Dermatol. 2014;71(4):725-30.

24. Johnson KE, Wilgus TA. Multiple roles for VEGF in non-melanoma skin cancer: angiogenesis and beyond. J Skin Cancer. 2012;2012:483439.

25. Argenziano G, Zalaudek I, Corona R, Sera F, Cicale L, Petrillo G, et al. Vascular structures in skin tumors: a dermoscopy study. Arch Dermatol. 2004;140(12):1485-9.

26. Boone MALM, Norrenberg S, Jemec GBE, Del Marmol V. High-definition optical coherence tomography imaging of melanocytic lesions: a pilot study. Arch Dermatol Res. 2014;306(1):11-26.
27. Ring HC, Mogensen M, Hussain AA, Steadman N, Banzhaf CA, Themstrup L, et al. Imaging of collagen deposition disorders using optical coherence tomography. J Eur Acad Dermatol Venereol. 2015;29(5):890-8.

28. Su P, Cao T, Tang MBY, Tey HL. In vivo high-definition optical coherence tomography: a bedside diagnostic aid for morphea. JAMA Dermatol. 2015;151(2):234-5.

29. Abignano G, Aydin SZ, Castillo-Gallego C, Liakouli V, Woods D, Meekings A, et al. Virtual skin biopsy by optical coherence tomography: the first quantitative imaging biomarker for scleroderma. Ann Rheum Dis. 2013;72(11):1845-51.

30. Ring HC, Themstrup L, Banzhaf CA, Jemec GBE, Mogensen M. Dynamic optical coherence tomography capillaroscopy: a new imaging tool in autoimmune connective tissue disease. JAMA Dermatol. 2016;152(10). doi:10.1001/jamadermatol. 2016.2027 (Epub ahead of print).

31. Paul DW, Ghassemi P, Ramella-Roman JC, Prindeze NJ, Moffatt LT, Alkhalil A, et al. Noninvasive imaging technologies for cutaneous wound assessment: a review. Wound Repair Regen. 2015;23(2):149-62.

32. Kuck M, Strese H, Alawi SA, Meinke MC, Fluhr JW, Burbach GJ, et al. Evaluation of optical coherence tomography as a non-invasive diagnostic tool in cutaneous wound healing. Skin Res Technol. 2014;20(1):1-7.

33. Park BH, Saxer CE, Srinivas SM, Nelson JS, de Boer JF. In vivo burn depth determination by high-speed fiber-based polarization sensitive optical coherence tomography. J Biomed Opt. 2001;6(4):474-9.

34. Srinivas SM, de Boer JF, Park BH, Keikhanzadeh K, Huang H-E, Zhang J, et al. Determination of burn depth by polarization-sensitive optical coherence tomography. J Biomed Opt. 2004;9(1):207-12.

35. Todorović M, Jiao S, Ai J, Pereda-Cubián D, Stoica $\mathrm{G}$, Wang LV. In vivo burn imaging using Mueller optical coherence tomography. Opt Express. 2008;16(14):10279-84.

36. Kim KH, Pierce MC, Maguluri G, Park BH, Yoon SJ, Lydon $M$, et al. In vivo imaging of human burn injuries with polarization-sensitive optical coherence tomography. J Biomed Opt. 2012;17(6):066012.

37. Gong P, Chin L, Es'haghian S, Liew YM, Wood FM, Sampson DD, et al. Imaging of skin birefringence for human scar assessment using polarization-sensitive optical coherence tomography aided by vascular masking. J Biomed Opt. 2014;19(12):126014. 
38. Fearmonti R, Bond J, Erdmann D, Levinson H. A review of scar scales and scar measuring devices. Eplasty. 2010;10:354-63.

39. Gong P, McLaughlin RA, Liew YM, Munro PRT, Wood FM, Sampson DD. Assessment of human burn scars with optical coherence tomography by imaging the attenuation coefficient of tissue after vascular masking. J Biomed Opt. 2014;19(2):21111.

40. Liew YM, McLaughlin RA, Gong P, Wood FM, Sampson DD. In vivo assessment of human burn scars through automated quantification of vascularity using optical coherence tomography. J Biomed Opt. 2013;18(6):061213.

41. Urban J, Siripunvarapon AH, Meekings A, Kalowitz A, Markowitz O. Optical coherence tomography imaging of erythematotelangiectatic rosacea during treatment with brimonidine topical gel $0.33 \%$ : a potential method for treatment outcome assessment. J Drugs Dermatol. 2014;13(7):821-6.

42. Themstrup L, Ciardo S, Manfredi M, Ulrich M, Pellacani G, Welzel J, et al. In vivo micro-morphological vascular changes induced by topical brimonidine studied by dynamic optical coherence tomography. J Eur Acad Dermatol Venereol. 2016;30(6):974-9.

43. Ring HC, Mogensen M, Banzhaf CA, Themstrup L, Jemec GBE. Optical coherence tomography imaging of teleangiectasias during intense pulsed light treatment: a potential tool for rapid outcome assessment. Arch Dermatol Res. 2013;305(4):299-303. 